\title{
The microstructure of a graphene-reinforced tennis racquet
}

\author{
Robert J. Young ${ }^{1} \cdot$ Mufeng Liu ${ }^{1}$
}

Received: 30 November 2015/Accepted: 16 December 2015/Published online: 8 January 2016

(C) The Author(s) 2016. This article is published with open access at Springerlink.com

\begin{abstract}
The microstructure of a graphene-reinforced tennis racquet has been analysed using a combination of optical microscopy and Raman spectroscopy. It is shown that the main structural components in the racquet frame are high-strength carbon fibres in an epoxy resin matrix. It is also found that graphene-based nanoparticles are used to reinforce resin-rich regions in the shaft of the racquet at the discontinuity in the fibre tows, where the handle is joined to the racquet head. From a detailed analysis of the relative positions and intensities of the Raman G and 2D bands, it is demonstrated that the nanoparticles employed in the racquet are most probably graphite nanoplatelets which have been added to improve the mechanical properties of the resin-rich regions. The nomenclature used for describing graphene-based materials is also discussed in the context of this present study.
\end{abstract}

\section{Introduction}

New high-performance materials are often first employed in sporting goods for a number of reasons. The main one is that people are prepared to pay a premium for the perceived sporting advantages that a new material may bring. Secondly, in many sporting applications, the product can be brought to market in a relatively short period of time with little need to verify its performance, in contrast to the lengthy period of testing that would be needed in, for

Robert J. Young

robert.young@manchester.ac.uk

1 School of Materials and National Graphene Institute, University of Manchester, Oxford Road, Manchester M13 9PL, UK example, aerospace certification. In addition, in most sporting applications, premature or unexpected failure of the component does not normally lead to catastrophic consequences. Thus, the sports that are most often used as a test-bed for these materials are typically, tennis, golf or winter sports such as skiing. This was certainly the case with carbon fibres being first used in tennis racquets in the early 1980s, some 25 years before they were introduced as main structural components in commercial aircraft such as the Boeing 787 and the A350 Airbus. Motor sport can also be an early user of new materials, although with a view to reducing the overall costs of the sport, new materials may even be banned by the regulations (e.g. carbon nanotubes are currently banned for use in composites in Formula One Motorsport [1]).

The study of graphene is one of the most exciting developments in recent years in materials science and condensed matter physics [2]. Graphene is thought to have good prospects for applications in a large number of different fields [3, 4]. There has been a rapid rise of interest in graphene following the first report in 2004 of the preparation and isolation of single graphene layers in Manchester [5]. Previously it had been thought that the isolation of single-layer graphene would not be possible since such 2D crystals were expected to be thermodynamically unstable and/or if prepared as single atomic layers could roll up into scrolls. This has certainly been shown not to be the case in the rapidly growing number of studies that have taken place since 2004. The initial excitement over graphene was because of its electronic properties. Its charge carriers exhibit very high intrinsic mobilities, having zero effective mass and are able, at room temperature, to travel distances of microns without being scattered [2]. Hence most of the original research upon graphene was concentrated upon its electronic properties, with that aim of 
applications such as using the material in electronic devices [6].

Graphene consists of a single atomic layer of $\mathrm{sp}^{2}$-hybridised carbon atoms arranged in a honeycomb structure. Research upon graphene expanded rapidly once it was realised that it could have other interesting physical properties, such as high levels of stiffness and strength, impermeability to gases and high levels of thermal conductivity. One clear application of graphene is in nanocomposites [7] and people working on different types of nanocomposites, such as the ones reinforced by nanoclays or nanotubes, rapidly changed their focus toward graphene-based nanocomposites. In addition, a number of people had worked previously upon the exfoliation of graphite into expanded graphite and on the oxidation of graphite into a poorly characterised material known as 'graphite oxide'. When the interest upon graphene grew, these materials were soon used to reinforce polymers once they were recognised as being different forms of graphene. Nanocomposites have now been prepared using a wide range of graphene-based materials in an increasing number of different polymers, and research upon these materials has been reviewed in detail elsewhere [7, 8].

This present report is concerned with the microstructural analysis of one of the first commercially produced graphene-based artefacts, i.e. a tennis racquet produced by the $\mathrm{HEAD}^{\circledR}$ company of Austria. It is presumed that the design of the racquet is based on the patent published by the company in 2013 [9]. According to the patent US2013/ 0090193, 'sporting goods may be designed to provide a user with a competitive, advantage, improve durability, enhance the user's comfort or protect the user from being injured. The marketability of sporting goods may depend on how effective they are at providing such benefits. As such, manufacturers of sporting goods continually seek to improve the materials and designs used in the construction of their products' [9]. It goes on to say that 'Thus, sporting goods are often times constructed of light weight, thin materials. However, if the materials are too thin or weak, they may lose their effectiveness, or may be easily damaged' [9]. It describes further that the state of the art in the construction and design of tennis racquets is to use fibrereinforced composite prepregs with a polymer resin matrix in the construction of the racquet frame. The main disclosure is that an improvement in performance of sporting goods such as tennis racquets may be further enhanced through the incorporation of graphene such that 'at least one prepreg layer may further include graphene material' [9]. A number of different forms of graphene are also described in the patent.

The aim of this paper is to undertake a microstructural analysis of a $\mathrm{HEAD}^{\circledR}$ tennis racquet and to employ a combination of optical microscopy and Raman spectroscopy to elucidate where the graphene has been used in, the type of graphene that has been employed and, if possible, to determine for what purpose. Raman spectroscopy has been employed for a number of years to characterise both structure and mechanical properties of carbon fibres [10-15] and is now used extensively to characterise different forms of graphene-based materials $[7,8,16,17]$.

\section{Nomenclature for graphene}

The nomenclature for graphene and a number of its derivatives has caused confusion and misunderstandings within the scientific community and there is a clear need for a better vocabulary for the nomenclature and standardisation of graphene. An important advance in this process was the paper of Bianco et al. [18], where each branch of the graphene family tree is described in detail. More recently, a similar paper by Wick et al. [19] also described clearly what is meant by the term graphene and the vocabulary for graphene-based materials. These two approaches need to be adopted more widely by the graphene community in order to avoid generalisations and unwanted complexity in the graphene literature. A helpful diagram on the classification of the different graphenebased forms can be found Fig. 1 taken from the work of Wick et al. [19], where the different materials are distinguished by the three fundamental characteristics.

The three fundamental properties which differentiate the different forms of graphene-based materials are the number of graphene layers, the average lateral flake dimensions and the atomic carbon/oxygen ratio [19]. On this basis, each graphene-based material presents different set of characteristics. Pristine graphene nanosheets are isolated, singleatom-thick sheets of hexagonally arranged, $\mathrm{sp}^{2}$-bonded carbon atoms. A few-layer graphene material is considered to consist of 2-5 sheets, while graphene material, consisting of 5-10 stacked graphene layers (sheets) with extended lateral dimension, can be termed as multilayer graphene. Above around 10 layers of sheets, the material is classified as graphite nanosheets or nanoplatelets depending upon the lateral dimensions. Chemically modified graphene is used widely as a reinforcing agent in polymer nanocomposites, particularly monolayer graphene oxide (GO) [20]. This originates from the chemical oxidation and exfoliation of graphite and is accompanied by extensive oxidative of the basal plane. The $\mathrm{C} / \mathrm{O}$ atomic ratio for as-produced $\mathrm{GO}$ is less than 3 and closer to 2 . Reduced graphene oxide (RGO) is a form of GO processed by chemical, thermal or other methods to reduce the oxygen content and so increase the $\mathrm{C} / \mathrm{O}$ ratio. The same classifications are used for $\mathrm{GO}$ with different numbers of layers as are used for graphene (Fig. 1). 


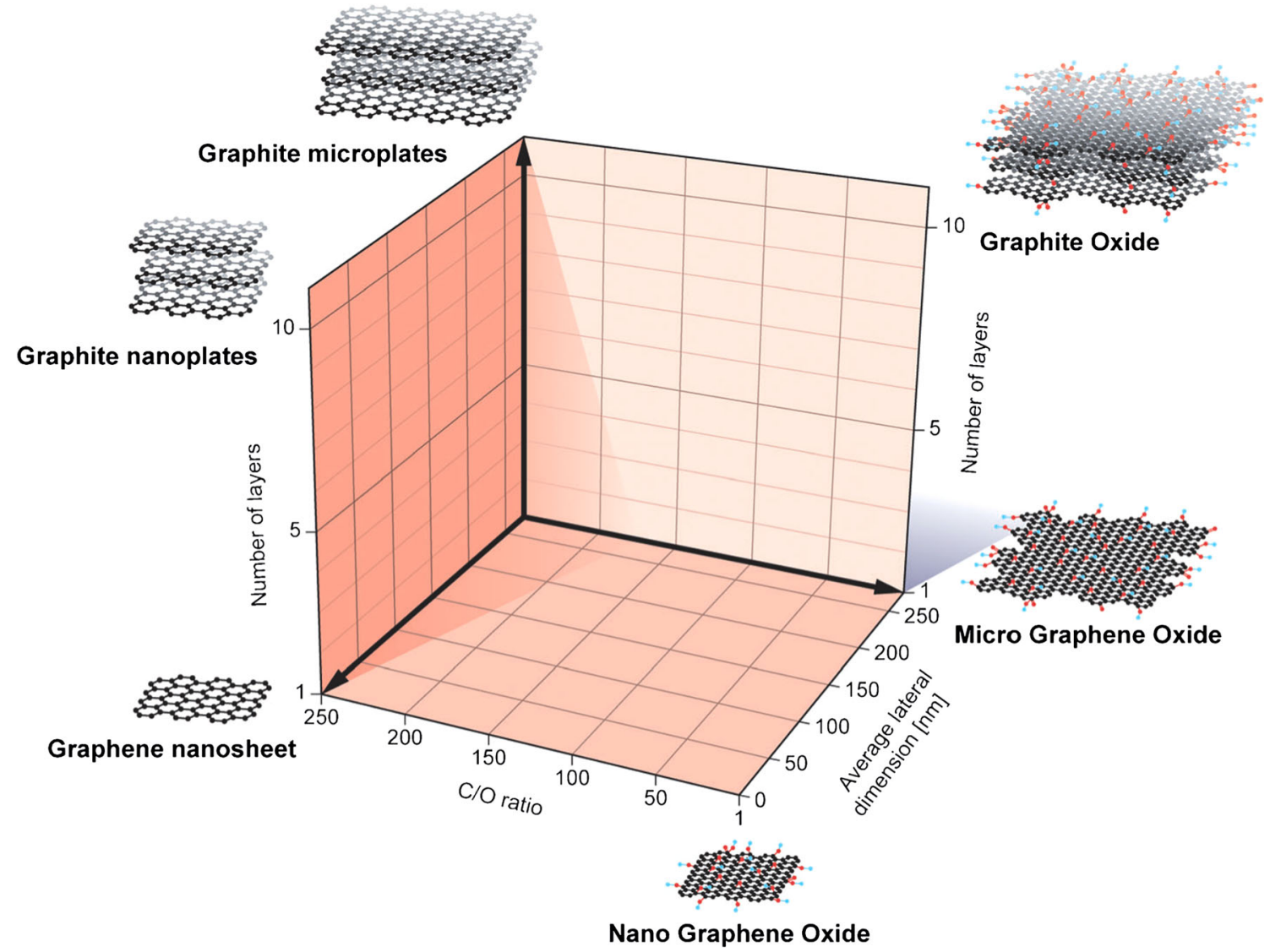

Fig. 1 Classification grid for the nomenclature of different graphene types, based on their fundamental properties. The materials drawn at the edges of the grid represent the ideal cases on the basis of the

\section{Experimental}

\section{Materials}

The artefact analysed in this study was the HEAD® Youtek Graphene Instinct Junior Tennis Racquet shown in Fig. 2. It has a weight of $240 \mathrm{~g}$, an overall length of $660 \mathrm{~mm}$ and head area of $240 \mathrm{~cm}^{2}$ and a string pattern of 16/19 (horizontal/longitudinal). For comparison purposes, Raman spectra were also obtained from mechanically exfoliated graphene monolayers [17, 21] and bilayers [22],

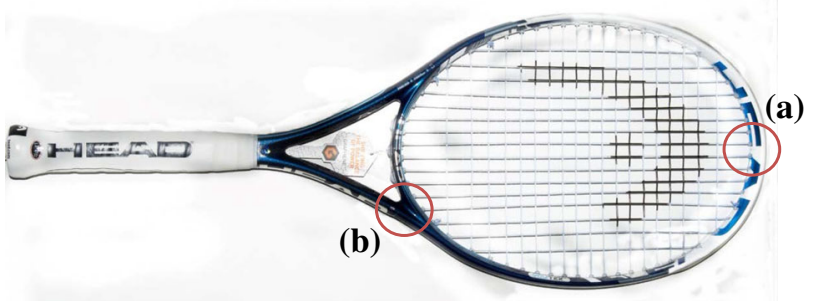

Fig. 2 The HEAD graphene tennis racquet showing the two regions investigated $a$ the tip and $b$ the shaft number of graphene layers, the $\mathrm{C} / \mathrm{O}$ ratio and the average lateral dimensions (reproduced with permission from [19] Copyright 2014, Wiley)

and M5 graphite nanoplatelets purchased from XG Sciences, Lansing, Michigan, USA. The nanoplatelets have an average diameter or width of about $5 \mu \mathrm{m}$ and a thickness of 6-8 nm [23].

\section{Specimen preparation}

Samples were prepared from the racquet for investigation by optical microscopy and Raman spectroscopy. Both the cross-sectional surfaces of the frame and surfaces along the axis of the frame were investigated. The racquet was firstly sawn into small sections (approximately $3-5 \mathrm{~cm}$ long) to fit into the cutting machine which was then used to slice the samples into specimens about $5 \mathrm{~mm}$ thick. The specimens were then set into polyester mounting resin and ground and polished to obtain smooth and flat surfaces. Considerable care had to be used to obtain surfaces free from grooves and scratches. Five levels of wet grinding paper were employed and the final paper used was a fine SiC P2500 grade paper with a grit size of $10 \mu \mathrm{m}$. The final stage of the specimen preparation was diamond polishing using a universal polisher and lubricant supplied by Buehler (ITW 
Test \& Measurement $\mathrm{GmbH}$ ), employing successively finer abrasive discs of 6,1 and $0.25 \mu \mathrm{m}$.

\section{Optical microscopy}

An Olympus $\mathrm{BH}-2$ series optical microscope was employed to analyse the microstructure of the polished sections using objective lenses of up to $50 \times$ magnification. Micrographs were obtained from the polished sections using the microscope CCD camera and saved in the form of jpeg files.

\section{Raman spectroscopy}

Raman spectra were obtained from the polished sections using a Renishaw 1000 Raman spectrometer system (Renishaw plc, UK) with a helium-neon laser of wavelength $633 \mathrm{~nm}$. The laser beam was concentrated on the sample with a $50 \times$ objective lens through another Olympus BH-2 optical microscope. The laser spot size was around $2 \mu \mathrm{m}$ in diameter. Raman spectra were obtained between 500 and $3200 \mathrm{~cm}^{-1}$. An exposure time of $10 \mathrm{~s}$ and an accumulation of up to 10 times were employed to reduce the noise. The software used to collect and analyse the data was Wire 3.3 by Renishaw. In order to determine Raman wavenumber and intensity of each peak accurately, Lorentzian curve fitting was employed.

\section{Results}

\section{Analysis of the racquet head}

The racquet head was found to have a hollow structure and polished sections of different areas of the head were examined by optical microscopy and it was found that up to 18 plies of prepreg were used in its construction. The plies were arranged such that fibres were aligned mainly tangentially around the racquet head.

Optical micrographs of transverse sections of the racquet head tip region (circled in Fig. 2) are presented in Fig. 3a, b showing cross sections of the fibres that were found to be of the order of $6 \mu \mathrm{m}$ in diameter. Figure $3 \mathrm{c}$ shows Raman spectra obtained from a fibre cross section and a region of resin between the plies. Since the laser spot size was of the order of $2 \mu \mathrm{m}$, spectra could be obtained from the separate components of the structure, free of interference from the adjacent material. The fibre spectrum shows broad $D$ and $G$ bands at around 1350 and $1590 \mathrm{~cm}^{-1}$, respectively, typical of polyacrylonitrile-based high-strength carbon fibres [15]. It should be noted that the 2D band, sometimes found at $2680 \mathrm{~cm}^{-1}$ in carbon fibres using HeNe laser excitation, is absent. This again is typical of high-strength carbon fibres and the 2D band is only found in more highly graphitised structures such as highmodulus carbon fibres [15] or graphene-based materials [7]. The Raman spectrum from the matrix resin between the plies, with a strong aromatic peak at around $1605 \mathrm{~cm}^{-1}$, is typical of the Raman spectrum found for epoxy resins [24]. Because of the limited amount of resin between the plies, the spectrum from the epoxy resin was found to be relatively weak with a low signal-to-noise ratio.

\section{Analysis of the shaft region}

Polished sections of different area of the racquet handle and shaft were also obtained in which there was a complex arrangement of fibres. A particular area of interest was the point at which the handle was connected to the racquet head. Optical micrographs of this area are shown in Fig. 4a, b. It can be seen that there are arrays of fibres aligned in different directions, typified by their oblique sections. Raman spectra obtained from these fibres were found to be similar to those of high-strength carbon fibres shown in Fig. 3c.

Figure $4 \mathrm{c}$ shows a Raman spectrum obtained from the large resin-rich region between the arrays of fibres. It can be seen that the spectrum is similar to that of the resin matrix shown in Fig. 3c with a better signal-to-noise ratio. There are, however, at least two extra Raman bands, indicated by arrows, present in the spectrum; a well-defined band at around $2680 \mathrm{~cm}^{-1}$ and another close to the main aromatic resin band at $1605 \mathrm{~cm}^{-1}$. The presence of these two extra bands is an indication of the presence of a second-phase in the epoxy resin matrix in this region.

\section{Discussion}

It is clear that the main structural elements in the tennis racquet are high-strength carbon fibres in an epoxy resin matrix. It has been shown, however, that there are resinrich regions in the area where the shaft of the racquet meets the racquet head and that extra Raman bands are found in these resin-rich regions. The microstructure of these resinrich regions was therefore examined further.

The Fig. 5a shows a high-magnification optical micrograph of the resin-rich region (along with a section of an isolated carbon fibre). Close inspection of the matrix microstructure shows that there is a speckled pattern of white particles with sub-micron dimensions in the epoxy matrix. The Raman spectrum from the resin-rich region was examined in detail and compared with that of the matrix region in the head of the racquet. Figure $5 \mathrm{~b}$ compares the Raman spectra from the two areas between 1500 

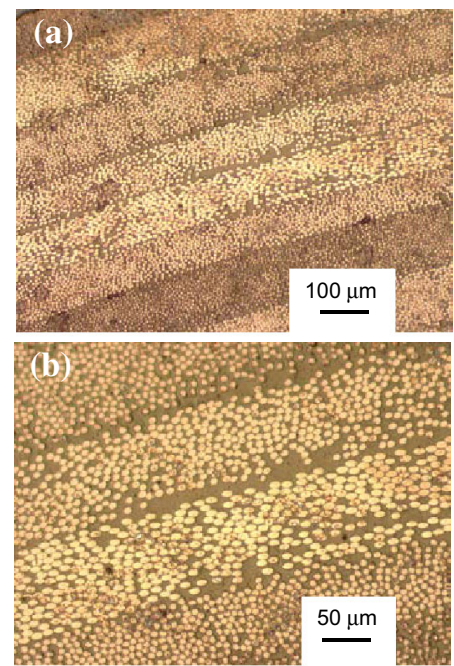

Fig. 3 The microstructure of the tip region of the racquet: a Optical micrograph of a transverse section of showing the fibres plies. b Optical micrograph of the same section at higher magnification

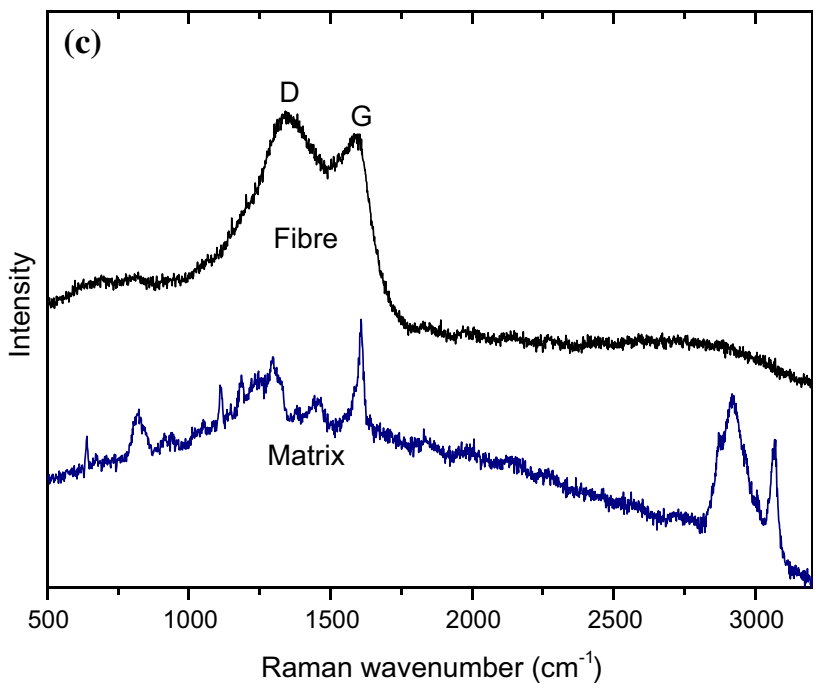

showing the resin-rich areas between the plies. $\mathbf{c}$ Raman spectra of the carbon fibres (showing the $D$ and $G$ bands) and the resin matrix
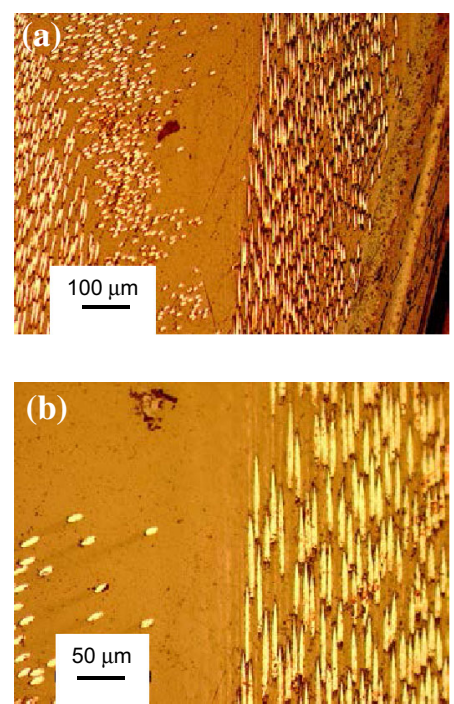

Fig. 4 The microstructure of the shaft region of the racquet: a Optical micrograph of a transverse section of showing a resin-rich region. b Optical micrograph of the same section at higher magnification.

and $1700 \mathrm{~cm}^{-1}$ and it can be seen that there is an extra narrow Raman band at $1580 \mathrm{~cm}^{-1}$ in the area where the particles are seen compared with the spectrum from the matrix in the head region. This band is clearly the G band from a graphite form of carbon [13, 15], but very different from the broad $\mathrm{G}$ bands obtained from the carbon fibres in the racquet (Fig. 3c). Figure 5c compares the Raman spectra between 2560 and $2760 \mathrm{~cm}^{-1}$ for the same two areas. In this case, it can be seen that there is an extra Raman band at around $2680 \mathrm{~cm}^{-1}$ when the particle are seen, which is not present at all in the epoxy matrix
(Fig. 3c). This extra band is characteristic of the 2D band in graphitic forms of carbon and again [15] is not found in the high-strength carbon fibres in the racquet (Fig. 3c).

It was concluded that the presence of the sub-micronsized particles and extra D and 2D Raman bands were an indication that some type of graphene-based material had been added to the resin-rich region of the racquet where the shaft meets the racquet head. In order to determine the exact form of graphene used, spectra in the 2D region were compared for different forms of graphene are shown in Fig. 6. It is known that the shape and position of the band 

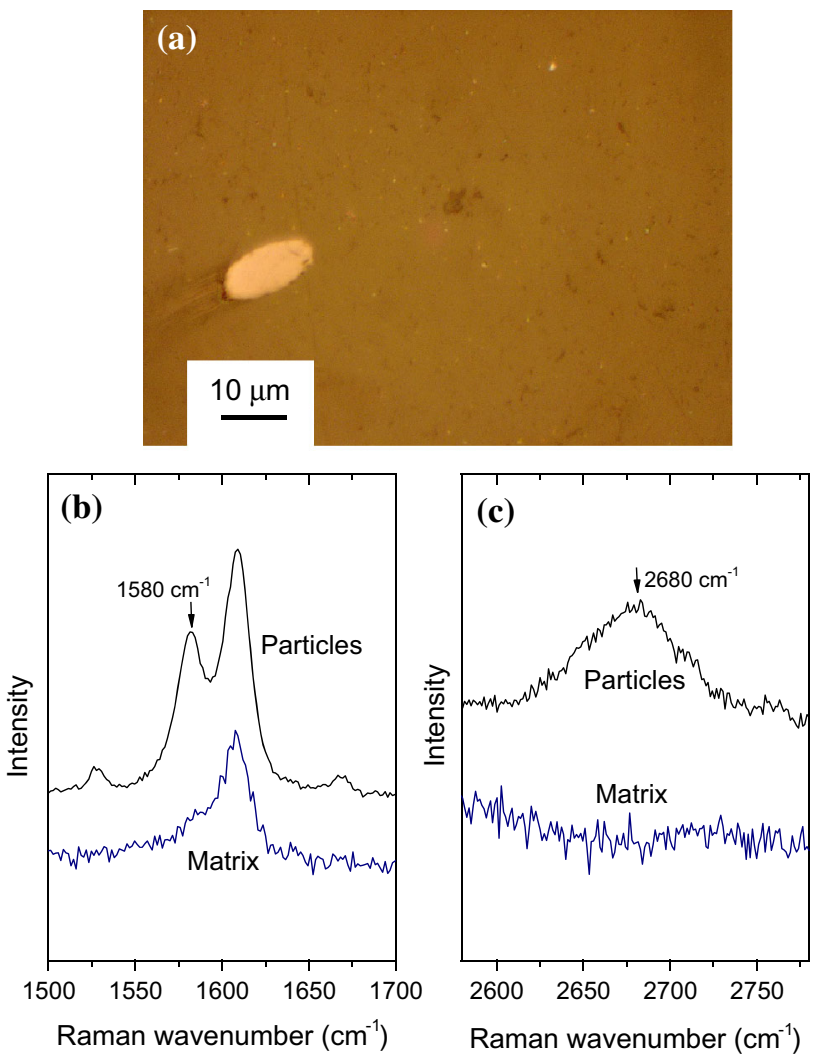

Fig. 5 a Optical micrograph of the resin-rich region of the racquet shaft with an isolated fibre showing the sub-micron-sized white particles in the resin. $\mathbf{b} \& \mathbf{c}$ Raman spectra of obtained from a region containing the small white particles

varies considerably with the type of graphene under investigation. In particular, the peak position of the $2 \mathrm{D}$ band increases as the number of layers in the graphene increases. With the $633 \mathrm{~nm}$ HeNe laser used, it can be seen from Fig. 6 that a sharp symmetrical 2D peak is found at around $2635 \mathrm{~cm}^{-1}$ for monolayer graphene, a broader more complex peak is found at about $2660 \mathrm{~cm}^{-1}$ for bilayer graphene and the M5 nanoplatelets with a thickness of 6-8 $\mathrm{nm}$ ( $\sim 25$ individual graphene layers thick) show a broad peak at around $2680 \mathrm{~cm}^{-1}$ with a lower wavenumber shoulder. Moreover it is found that the exact position of the band for each particular form of graphene varies with stress when the material is deformed, for example, in composite matrix [22]. This phenomenon can be used to follow the micromechanics of the deformation of different forms of graphene [20, 21].

The shape and position of the 2D band from the particles in the resin-rich region (Fig. 5c) is most similar to that of the nanoplatelets in Fig. 6. This implies that graphene nanoplatelets, or more correctly, graphite nanoplatelets (Fig. 1), have been added to the epoxy resin in the resinrich regions of the area where the head is joined onto the racquet handle. This resin-rich region is clearly a potential

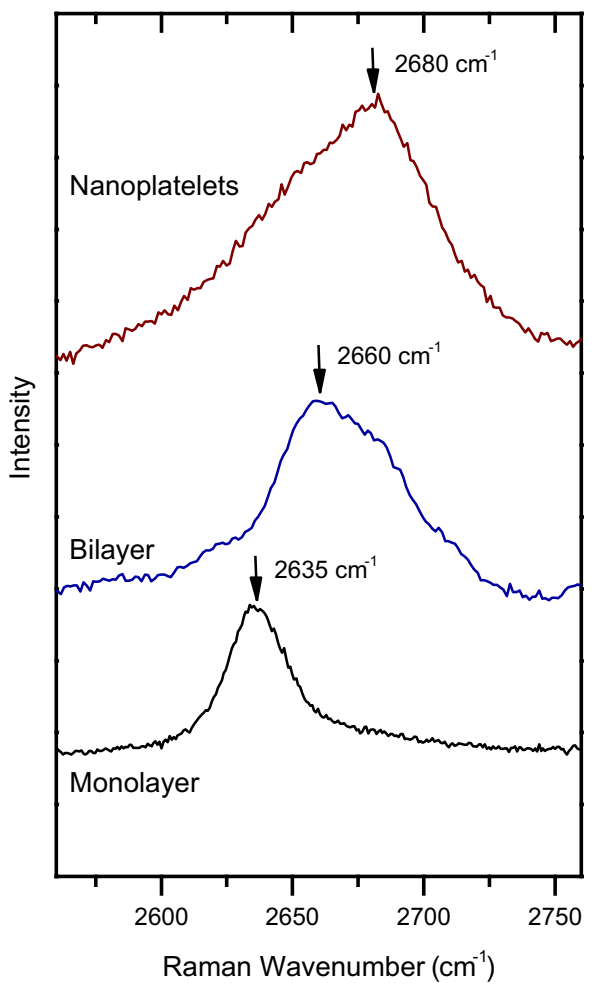

Fig. 6 Differences in the shape and position of the 2D Raman band for different forms of graphene: monolayer, bilayer and XG graphite nanoplatelets

point of weakness in the racquet during use, and it is likely that the addition of the nanomaterial is aimed at improving the mechanical performance of the component [7]. It is well established that the addition of graphene-based materials can increase both the stiffness [25] and toughness [26] of epoxy resins. It appears therefore that the graphite nanoplatelets have been added to the resin-rich region of the racquet to improve the mechanical properties in this area which might otherwise be a point of potential weakness. It was not possible, however, to evaluate the effect of the addition of the graphite nanoplatelets to the resin-rich region in the absence of equivalent samples without graphite nanoplatelets added.

\section{Conclusions}

It has been shown that the combination of optical microscopy and Raman spectroscopy is a powerful method of characterising the microstructure of a graphene-based tennis racquet. It has been found that the main structural component in the racquet is high-strength carbon fibres in an epoxy resin matrix. Resin-rich regions have been found in the area where the head of the racquet is joined to the handle. It appears that this area, which is a point of 
potential of weakness in the racquet, has been reinforced with graphene in form of graphite nanoplatelets.

Acknowledgements This research has been supported by funding from the European Union Seventh Framework Programme under grant agreement No 604391, the Graphene Flagship.

\section{Compliance with ethical standards}

Conflicts of interest The authors have no conflict of interest associated with this study and do not wish to offer any endorsement of the product under analysis.

Open Access This article is distributed under the terms of the Creative Commons Attribution 4.0 International License (http://crea tivecommons.org/licenses/by/4.0/), which permits unrestricted use, distribution, and reproduction in any medium, provided you give appropriate credit to the original author(s) and the source, provide a link to the Creative Commons license, and indicate if changes were made.

\section{References}

1. Formula One Technical Regulations (2015) Federation Internationale de L'Automobile (FiA), Paris

2. Geim AK, Novoselov KS (2007) The rise of graphene. Nat Mater 6:183-191. doi:10.1038/Nmat1849

3. Geim AK (2011) Nobel lecture: random walk to graphene. Rev Mod Phys 83:851-862. doi:10.1103/RevModPhys.83.851

4. Novoselov KS (2011) Nobel lecture: graphene: materials in the flatland. Rev Mod Phys 83:837-849. doi:10.1103/RevModPhys. 83.837

5. Novoselov KS, Geim AK, Morozov SV et al (2004) Electric field effect in atomically thin carbon films. Science 306:666-669. doi:10.1126/science.1102896

6. Avouris P (2010) Graphene: electronic and photonic properties and devices. Nano Lett 10:4285-4294. doi:10.1021/nl102824h

7. Young RJ, Kinloch IA, Gong L, Novoselov KS (2012) The mechanics of graphene nanocomposites: a review. Compos Sci Technol 72:1459-1476. doi:10.1016/j.compscitech.2012.05.005

8. Papageorgiou DG, Kinloch IA, Young RJ (2015) Graphene/ elastomer nanocomposites. Carbon 95:460-484. doi:10.1016/j. carbon.2015.08.055

9. HEAD Technology GMBH, Ltd (2013) US Patent 2013/0090193, USA

10. Gu XH, Young RJ, Day RJ (1995) Deformation micromechanics in model carbon-fiber-reinforced composites 1 . The single-fiber pullout test. J Mater Sci 30:1409-1419. doi:10.1007/Bf00375240
11. Huang YL, Young RJ (1994) Microstructure and mechanicalproperties of pitch-based carbon-fibers. J Mater Sci 29:4027-4036. doi:10.1007/Bf00355965

12. Kong K, Deng LB, Kinloch IA, Young RJ, Eichhorn SJ (2012) Production of carbon fibres from a pyrolysed and graphitised liquid crystalline cellulose fibre precursor. J Mater Sci 47:5402-5410. doi:10.1007/s10853-012-6426-y

13. Tanaka F, Okabe T, Okuda $H$ et al (2013) The effect of nanostructure upon the deformation micromechanics of carbon fibres. Carbon 52:372-378. doi:10.1016/j.carbon.2012.09.047

14. Tanaka F, Okabe T, Okuda H, Kinloch IA, Young RJ (2013) The effect of nanostructure upon the compressive strength of carbon fibres. J Mater Sci 48:2104-2110. doi:10.1007/s10853-012-6984-z

15. Young RJ (1995) Monitoring deformation processes in highperformance fibres using Raman spectroscopy. J Text Inst 86:360-381. doi:10.1080/00405009508631340

16. Gong L, Young RJ, Kinloch IA et al (2013) Reversible loss of bernal stacking during the deformation of few-layer graphene in nanocomposites. ACS Nano 7:7287-7294. doi:10.1021/nn402830f

17. Young RJ, Gong L, Kinloch IA, Riaz I, Jalil R, Novoselov KS (2011) Strain mapping in a graphene monolayer nanocomposite. ACS Nano 5:3079-3084. doi:10.1021/nn2002079

18. Bianco A, Cheng HM, Enoki T et al (2013) All in the graphene family - a recommended nomenclature for two-dimensional carbon materials. Carbon 65:1-6. doi:10.1016/j.carbon.2013.08.038

19. Wick P, Louw-Gaume AE, Kucki M et al (2014) Classification framework for graphene-based materials. Angew Chem Int Ed 53:7714-7718. doi:10.1002/anie.201403335

20. Li ZL, Young RJ, Kinloch IA (2013) Interfacial stress transfer in graphene oxide nanocomposites. ACS Appl Mater Interfaces 5:456-463. doi:10.1021/am3025131e

21. Gong L, Kinloch IA, Young RJ, Riaz I, Jalil R, Novoselov KS (2010) Interfacial stress transfer in a graphene monolayer nanocomposite. Adv Mater 22:2694-2697. doi:10.1002/adma. 200904264

22. Gong L, Young RJ, Kinloch IA, Riaz I, Jalil R, Novoselov KS (2012) Optimizing the reinforcement of polymer-based nanocomposites by graphene. ACS Nano 6:2086-2095. doi:10. $1021 / \mathrm{nn} 203917 \mathrm{~d}$

23. Technical data sheet $-\mathrm{xGnP} \circledast$ graphene nanoplatelets (2013) XG sciences Inc., Lansing, Michigan, USA

24. Chike KE, Myrick ML, Lyon RE, Angel SM (1993) Raman and near-infrared studies of an epoxy-resin. Appl Spectrosc 47:1631-1635. doi:10.1366/0003702934334714

25. Li Z, Wang RG, Young RJ et al (2013) Control of the functionality of graphene oxide for its in epoxy nanocomposites application. Polymer 54:6437-6446. doi:10.1016/j.polymer.2013.09.054

26. Li Z, Young RJ, Wang RG et al (2013) The role of functional groups on graphene oxide in epoxy nanocomposites. Polymer 54:5821-5829. doi:10.1016/j.polymer.2013.08.026 